\title{
Efecto de la Temperatura en la Evaluación de la Fatiga en Ligantes Asfálticos
}

\section{Effect of temperature on fatigue evaluation of asphalt binders}

\section{M.I. Yelitza Ayala del Toro}

Universidad Autónoma de Chihuahua-Facultad de Ingeniería, México

yelitzaayala@hotmail.com

\section{Dr. Paul Garnica Anguas}

Instituto Mexicano del Transporte-Laboratorio de Infraestructura,

México

pgarnica@imt.mx
Dr. Horacio Delgado Alamilla

Instituto Mexicano del Transporte-Laboratorio de Infraestructura, México.

hdelgado@imt.mx

Fecha de recepción: 18 de noviembre de 2015 / Fecha de aprobación: 07 de abril de 2016

\section{RESUMEN}

La fatiga es uno de los modos de falla más comunes en los pavimentos flexibles, la cual es caracterizada por la interconexión de grietas longitudinales y transversales provocadas por los esfuerzos de tensión que se generan en la base de la carpeta asfáltica, debido al tránsito pesado.

El presente trabajo evalúa la influencia de la temperatura en el comportamiento a la fatiga de ligantes asfálticos, de acuerdo a dos métodos de ensayo: Grado PG y Barrido de Amplitud Lineal (LAS) (ASTM D7175, 2008). El estudio evalúa tres ligantes asfálticos, los cuales corresponden a tres diferentes aplicaciones de la mezcla asfáltica: PG6416 (mezcla convencional), PG76-16 (mezcla modificada con polímero SBS) y PG88-22 (mezcla tibia). Los resultados obtenidos muestran la diferencia en comportamiento de los ligantes cuando son evaluados a distintas temperaturas $\left(37,34,28\right.$, y $\left.20^{\circ} \mathrm{C}\right)$ considerando dos tipos de envejecimiento: a corto plazo y a largo plazo. Adicionalmente, se explora la temperatura de transición entre la parte sólido-elástica y la líquido-viscosa, como parámetro para determinar la temperatura adecuada del ensayo de fatiga.

Los resultados obtenidos indican que existe un cambio en la tendencia de la resistencia a fatiga con el cambio de temperatura, principalmente cuando el asfalto se encuentra a una temperatura superior o inferior al punto (temperatura) de transición. De acuerdo a la evaluación por fatiga de la mezcla asfáltica, la temperatura de transición indica ser un parámetro adecuado para la selección de la temperatura de evaluación de este modo de falla en ligantes asfálticos; sin embargo, se deberán realizar validaciones posteriores con diferentes materiales para corroborarlo.

PALABRAS CLAVES: ligante asfáltico, reología, fatiga, LAS, 4PBB

\section{ABSTRACT}

The fatigue cracking is one of the most common distresses in flexible pavements, which is characterized by the connection of longitudinal and transverse cracks caused by the tensile stresses generated in the base of the asphalt layer, produced by the heavy traffic loads.

This study evaluates the influence of temperature on fatigue failure in asphalt binders, according to two test methods: PG Grade and the Linear Amplitude Sweep (LAS) test. The research evaluates three asphalt binders, which correspond to three different applications in the asphalt mix: PG64-16 (conventional mix), PG76-16 (modified mix with polymer SBS) and PG88-22 (warm mix). The results show the difference in behavior of the binders when evaluated at different temperatures $\left(37,34,28\right.$, and $20^{\circ} \mathrm{C}$ ) considering two types of aging: short and long term. Additionally, the transition temperature between the solid-elastic and the liquid-viscous part of the binder is explored, as a parameter for selecting a suitable temperature for the fatigue test.

The results indicate a variation in the trend of the binder with most resistance to fatigue failure, with the change in temperature, particularly when the asphalt is subjected to temperatures above or below its transition point (temperature). According to the fatigue evaluation in the asphalt mixture, the transition temperature seems to be a good criteria to select an adequate temperature to evaluate fatigue in asphalt binders; nevertheless, additional validations with different materials must be done to support the results.

KEY WORDS: asphalt binder, rheology, fatigue, LAS, $4 P B B$ 


\section{INTRODUCCIÓN}

Los pavimentos asfálticos están sometidos a diversas solicitaciones mecánicas y ambientales, las cuales producen degradaciones en los mismos. Una de las degradaciones más comunes en los pavimentos es la fisuración por fatiga. Existen diferentes ensayos de laboratorio para realizar su evaluación en la mezcla asfáltica, sin embargo dichos ensayos consumen muchos recursos (tiempo y costo) (Ghuzlan et al., 2003). Por este motivo, en los últimos años se han tratado de desarrollar ensayos que permitan predecir el comportamiento de la mezcla asfáltica a partir de la evaluación del ligante asfáltico (ASTM D 6373, 2008, Bahía et al., 2001, Hintz et al., 2013; Van et al.,2012).

Muchos investigadores creen que la fatiga es un problema de la estructura del pavimento, mientras que otros creen que es un problema de la mezcla asfáltica. Sin embargo, es reconocido entre ambos grupos que la grieta por fatiga comienza y se propaga en el asfalto; por lo tanto su estudio debe tomarse en cuenta desde el ligante asfáltico (Bahía et al., 2001).

En el Reporte 459 del Programa de Investigación de la Cooperativa Nacional de Carreteras (NCHRP), se concluyó que la actual especificación "Grado PG" - para caracterización de ligantes asfálticos - generalmente subestima el desempeño potencial de los mismos, particularmente los modificados, ya que se evalúan únicamente en el rango viscoelástico lineal. Por este motivo, se desarrolló un método para evaluar la fatiga en ligantes asfálticos realizando un barrido de tiempo (time sweep test) llamado Carga Cíclica Repetida (RCL, del inglés Repeated Cyclic Loading) (Bahía et al., 2001).

Delgado et al. (2015) concluyen que el ensayo RCL efectivamente captura la respuesta viscoelástica no lineal de los ligantes asfálticos; sin embargo, es un ensayo que consume muchos recursos (aprox. 16,000 ciclos, $f=10 \mathrm{~Hz}$ ). Por este motivo, los autores realizaron una comparativa del ensayo RCL con el ensayo Barrido de Amplitud Lineal (LAS, del inglés Linear Amplitude Sweep), donde se concluye que el método LAS es congruente con el RCL, además de obtener la ley de fatiga completa en una sola prueba.

Debido a que el asfalto es un material termo-sensible, un aspecto importante a considerar en la evaluación de la fatiga es la temperatura del ensayo (Hossein et al., 2003). Sin embargo los ensayos propuestos no definen con claridad la temperatura adecuada para la evaluación de la fatiga en el ligante asfáltico, por lo cual es indispensable evaluar la influencia de este parámetro en los ensayos de predicción analizados.

Un material viscoelástico puede pasar de un estado líquidoviscoso a un estado sólido-elástico, por medio de la temperatura; por lo tanto, hace falta un parámetro que defina la temperatura de ensayo considerando este cambio de fase del material.

El presente estudio tiene como objetivo principal evaluar la influencia de la temperatura en la predicción del comportamiento a la fatiga de tres ligantes asfálticos considerando dos ensayos (Grado PG y LAS), así como la pertinencia de implementación en México del ensayo LAS.

\section{MÉTODOS DE ENSAYO}

Existen distintos ensayos para evaluar la fatiga en ligantes asfálticos, con la intención de predecir el comportamiento de una mezcla asfáltica a este modo de falla. Los ensayos más utilizados son: el ensayo de fatiga avanzado propuesto por la ENTE/DGCP (Van et al.,2012), el ensayo RCL propuesto por Bahia (2001), el ensayo LAS propuesto por el Centro de Investigación de Asfalto Modificado de la Universidad de Wisconsin (Hintz et al., 2001), y el Grado PG (ASTM D6373), del método Superpave. En la presente investigación se compararán los métodos del Grado PG y LAS, a distintas temperaturas.

\section{Grado PG}

Este ensayo se realiza de acuerdo a la norma ASTM D7175 (2008), el cual es ejecutado en el reómetro de corte dinámico (DSR); tiene la finalidad de evaluar la resistencia a la fatiga del ligante asfáltico. El procedimiento de prueba consiste en aplicar un esfuerzo sinusoidal a una muestra de asfalto - envejecida previamentede $8 \mathrm{~mm}$ de diámetro y $2 \mathrm{~mm}$ de espesor, mediante dos platos paralelos. El equipo aplica el esfuerzo necesario para lograr que el material presente un $1 \%$ de deformación a una frecuencia de 10 $\mathrm{rad} / \mathrm{s}$. Se realiza un barrido de temperaturas en el rango intermedio de temperatura $\left(4-40{ }^{\circ} \mathrm{C}\right)$ y se determina la temperatura mínima de resistencia a la fatiga cuando el parámetro de fatiga, $G *(\operatorname{sen} \delta)$, es menor o igual a $5,000 \mathrm{kPa}$.

La caracterización por Grado PG involucra dos tipos de envejecimientos: uno a corto plazo conocido como RTFO (Horno Rotatorio de Película Delgada, del inglés Rolling Thin Film Oven), y uno a largo plazo conocido como PAV (Vasija de Envejecimiento a Presión, de inglés Pressure Aging Vessel). La evaluación por fatiga en el Grado PG se especifica utilizando asfalto residuo de PAV.

\section{Barrido de Amplitud Lineal (LAS)}

El ensayo LAS se lleva a cabo de acuerdo al método AASHTO TP101 (2014), el cual determina la resistencia al daño por fatiga por medio de una carga cíclica (sinusoidal) e incrementando la amplitud de carga en forma lineal; la intención del ensayo es causar un daño acelerado por fatiga. El enfoque de Daño Continuo 
se utiliza para calcular la resistencia a la fatiga a partir de las propiedades reológicas del ligante y los resultados del barrido de amplitud.

El ensayo se realiza en el (DSR) de acuerdo a los parámetros mostrados en la Tabla 1, donde se divide en 2 etapas, siendo la primera un barrido de frecuencias a una deformación constante (0.1\%); mientras que la segunda es un barrido de deformaciones a frecuencia constante $(10 \mathrm{~Hz})$. Esto permite realizar el análisis del Daño Continuo Viscoelástico (VECD), donde se calcula el parámetro de desempeño a fatiga del ligante, $\mathrm{N}_{\mathrm{f}}$.

Tabla 1. Generalidades del ensayo LAS

\begin{tabular}{|c|c|}
\hline Origen de la muestra & Residuo de RTFO o PAV \\
\hline $\begin{array}{c}\text { Temperatura de ensayo } \\
\text { Geometria / Espesor }\end{array}$ & Intermedia de ASTM D6373 \\
\hline \multicolumn{2}{|c|}{$8 \mathrm{~mm} / 2 \mathrm{~mm}$} \\
\hline $\begin{array}{c}\text { Deformación (\%)/ } \\
\text { Frecuencia (Hz) }\end{array}$ & 0.1 / barrido de $0.2-30$ \\
\hline \multicolumn{2}{|c|}{ Etapa 2 } \\
\hline $\begin{array}{c}\text { Frecuencia (Hz)/ } \\
\text { Deformación (\%) }\end{array}$ & $10 /$ barrido de 0 - 30 \\
\hline Ciclos requeridos & 3,100 \\
\hline
\end{tabular}

Fuente: Elaboración propia a partir de AASHTO TP101 (2014)

El análisis de la fatiga por medio de este ensayo involucra generar la curva de Wöhler; la cual es una curva representativa que permite visualizar los materiales en el rango de fatiga. Define una relación entre la solicitación (esfuerzo o deformación) y el número de ciclos a la ruptura, $\mathrm{N}_{\mathrm{R}}$.

\section{EVALUACIÓN DE LA FATIGA}

\section{Descripción de los materiales}

Se evaluaron tres cementos asfálticos comerciales distintos, para diferentes aplicaciones, los cuales fueron clasificados de acuerdo a su Grado PG con la norma ASTM D 6373 como se indica en la Tabla 2.

\section{Tabla 2. Clasificación por Grado PG de asfaltos}

\begin{tabular}{|c|c|c|c|}
\hline Grado PG & Nomenclatura & Aplicación & Modificador \\
\hline PG 64-16 & PG 64 & $\begin{array}{c}\text { Mezcla } \\
\text { convencional }\end{array}$ & Sin modificador \\
\hline PG 76-16 & PG 76 & $\begin{array}{l}\text { Mezcla con asfalto } \\
\text { modificado }\end{array}$ & Polímero SBS \\
\hline PG 88-22 & PG 88 & Mezcla tibia & Aminas grasas \\
\hline
\end{tabular}

\section{Evaluación por Grado PG}

La temperatura intermedia calculada para cada ligante asfáltico se indica en la Tabla 3, la cual se obtiene de la ASTM D6373 mediante las temperaturas alta y baja del Grado PG. Por otro lado también se indica la temperatura intermedia medida, la cual se refiere a la temperatura donde el parámetro $\mathrm{G}^{*}(\operatorname{sen} \delta)$ es igual a 5,000 kPa.

Tabla 3. Resistencia a fatiga - Residuo de PAV

\begin{tabular}{|c|c|c|c|}
\hline Grado PG & $\begin{array}{c}\text { Temperatura } \\
\text { calculada (ASTM } \\
\text { D6373) }\end{array}$ & $\begin{array}{c}\mathrm{G}^{*} \operatorname{sen} \delta(\mathrm{kPa}) \\
\text { @ T calculada }\end{array}$ & $\begin{array}{r}\text { Temperatura } \\
\text { medida } \\
\left(\mathrm{G}^{*} \operatorname{sen} \delta=\right. \\
5000 \mathrm{kPa})\end{array}$ \\
\hline PG 64-16 & $28^{\circ} \mathrm{C}$ & 3,056 & $22.8^{\circ} \mathrm{C}$ \\
\hline PG 76-16 & $34^{\circ} \mathrm{C}$ & 1,084 & $19.6{ }^{\circ} \mathrm{C}$ \\
\hline PG 88-22 & $37^{\circ} \mathrm{C}$ & 565 & $18.4{ }^{\circ} \mathrm{C}$ \\
\hline
\end{tabular}

De acuerdo a la temperatura intermedia calculada del pavimento y a las rigideces obtenidas, el asfalto PG 88 indica tener un mejor comportamiento a la fatiga, debido a que presenta una menor rigidez a las temperaturas evaluadas (Tabla 4). Por lo que la metodología Grado PG establece que el asfalto PG88 tiene el mejor comportamiento a la fatiga.

\section{Tabla 4. Resistencia a temperaturas intermedias}

\begin{tabular}{|c|c|c|c|}
\hline \multirow{2}{*}{ Ligante } & \multicolumn{3}{|c|}{$\mathrm{G}^{*} \operatorname{sen} \delta(\mathrm{kPa}) @$ Tcalculada } \\
\cline { 2 - 4 } & $28^{\circ} \mathrm{C}$ & $34^{\circ} \mathrm{C}$ & $37^{\circ} \mathrm{C}$ \\
\hline PG 64-16 & 3,056 & 1,637 & 1,053 \\
\hline PG 76-16 & 2,096 & 1,084 & 973 \\
\hline PG 88-22 & 1,646 & 788.5 & 565 \\
\hline
\end{tabular}

\section{Evaluación con el ensayo LAS}

Como lo indica el método de prueba, se evaluaron los ligantes envejecidos en RTFO a las temperaturas intermedias para obtener resultados comparables entre ellos y a la vez observar su comportamiento a distintas temperaturas. Los resultados se muestran en la Figura 1.

En esta Figura se observó primeramente que el ligante PG 88 presenta el mejor comportamiento a la fatiga y que la tendencia es la misma a las tres temperaturas evaluadas. Esto es un indicativo preliminar de que la resistencia a fatiga evaluada con el ensayo LAS es congruente con la del Grado PG.

Para la temperatura más alta $\left(37{ }^{\circ} \mathrm{C}\right)$ se visualiza un comportamiento casi lineal entre el esfuerzo y la deformación — sin importar la amplitud de la solicitación- no se observa un punto de falla en la curva. Este comportamiento se genera debido a que los ligantes se encuentran en un estado líquido viscoelástico, por lo que no se presenta el fenómeno de fatiga. Por el contrario, conforme se disminuyó la temperatura, se presentó el fenómeno de falla. 

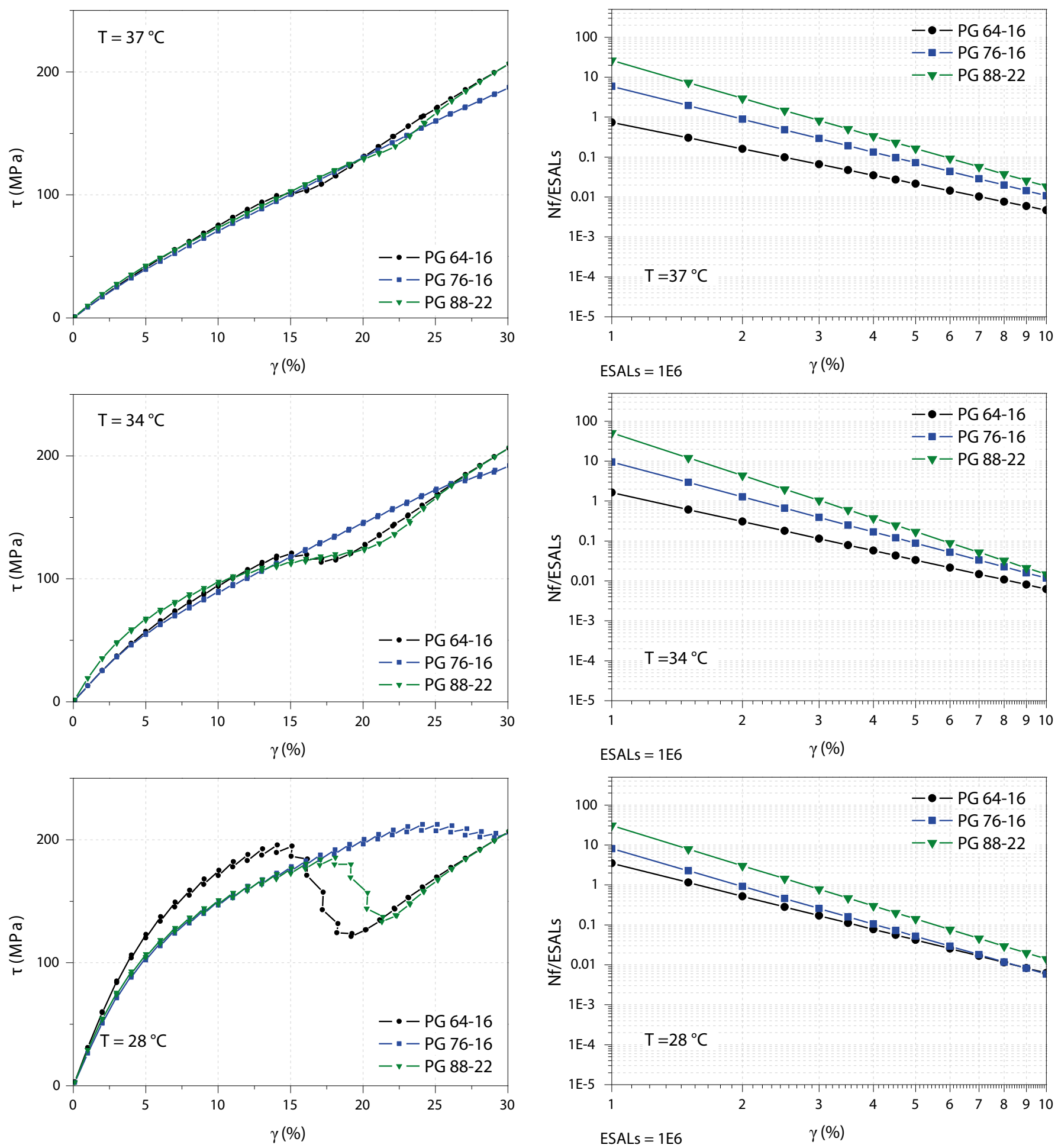

Figura 1. Resultados LAS a 37,34 y $28^{\circ} \mathrm{C}$; RTFO

Aunado a la evaluación de los ligantes a la temperatura intermedia del pavimento, se decidió evaluarlos también a $20^{\circ} \mathrm{C}$ con el objetivo de verificar la tendencia del comportamiento a una temperatura cercana a la temperatura promedio medida del parámetro de fatiga $\left(G^{*}\right.$ sen $\left.\delta\right)$, indicadas en la Tabla 3. Cabe señalar que es a esta temperatura que se realizan los ensayos de fatiga en la mezcla asfáltica en México. Los resultados obtenidos se observan en la Figura 2. 

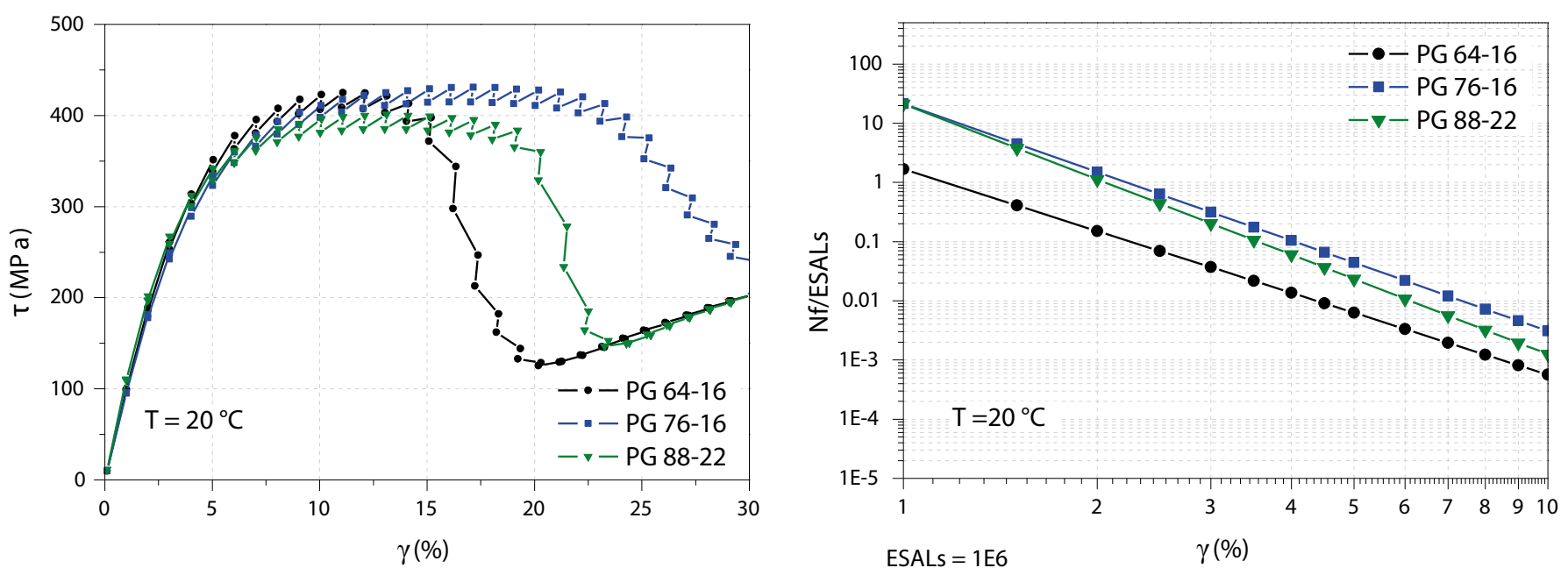

Figura 2. Resultados LAS a $20^{\circ} \mathrm{C}$, RTFO

Observando los resultados obtenidos a una temperatura de $20^{\circ} \mathrm{C}$, se detectó una discrepancia con los resultados evaluados a las temperaturas intermedias calculadas. La tendencia cambió, siendo para esta temperatura el ligante PG 76 el que presenta un mejor comportamiento a la fatiga.

El cambio en la tendencia del ligante con mayor resistencia a la fatiga de acuerdo a las distintas temperaturas $\left(37,34\right.$ y $28{ }^{\circ} \mathrm{C}$ vs $20{ }^{\circ} \mathrm{C}$ ), se puede atribuir a la transición de un comportamiento sólido-elástico a un líquido-viscoso en el material. Este punto de transición será definido por el equilibrio entre la parte elástica $\left(G^{\prime}\right)$ y viscosa (G”) del material, el cual se establece como el ángulo de fase igual a $45^{\circ}$; donde para valores superiores de $45^{\circ}$ el comportamiento mecánico estará regido por la parte viscosa del material; mientras que para valores inferiores, dominará la parte elástica.

\section{TEMPERATURA DE TRANSICIÓN}

La temperatura de transición se empleará para denominar la temperatura a la que se produce la transición de fase del estado sólido-elástico a líquido-viscoso. En la Figura 3, se observa cómo la temperatura de transición hacia la izquierda (mayor temperatura), el asfalto presentaría un comportamiento líquido viscoelástico hasta comportarse como un líquido viscoso. Por otro lado, desplazándose a la derecha (menor temperatura), el asfalto presentaría un comportamiento sólido viscoelástico hasta llegar a comportarse como un sólido rígido.

Los problemas de fisuración de una carpeta asfáltica, se presentarán precisamente debajo de la temperatura de transición, donde el ligante comenzará a comportarse como un sólido y será más propenso a presentar agrietamiento. Cuando el asfalto es

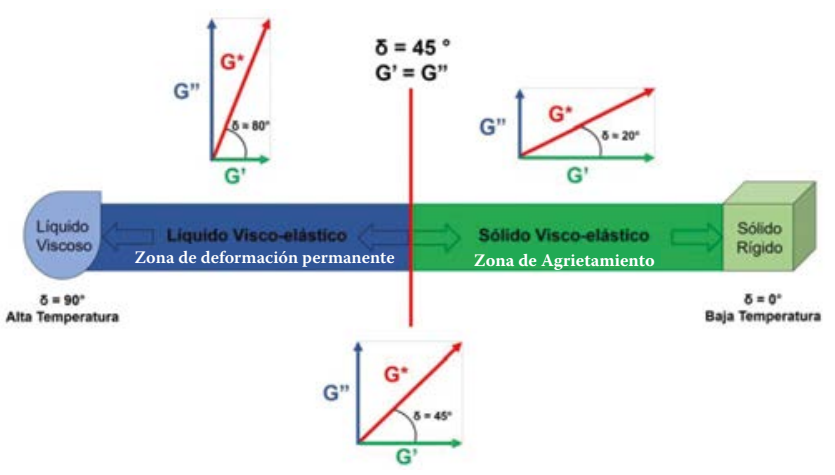

Figura 3. Temperatura de transición en ligantes asfálticos. Gutiérrez (2013)

sometido a un esfuerzo, la componente elástica (G') absorbe la energía y la utiliza para recuperarse; en cambio, la componente viscosa (G") disipa la energía ya sea por medio de calor o por deformación del material. Gutiérrez (2013) afirma que:

El estado ideal del asfalto es cuando estas componentes son iguales en magnitud $\left(\delta=45^{\circ}\right)$, ya que la componente elástica absorbe sólo la energía necesaria para recuperarse de la deformación sufrida por el esfuerzo y libera la energía que no necesita por medio de la componente viscosa.

De acuerdo a lo anterior, el autor recomienda evaluar las propiedades a la fatiga a una temperatura menor o igual a la temperatura de transición del ligante asfaltico. Por este motivo, se determinó el punto de transición para cada ligante asfáltico en sus dos condiciones de envejecimiento: RTFO y PAV.

En la Figura 4 se puede observar la variación del ángulo de fase ( $\delta)$ con la temperatura, determinando así la temperatura a la que el ángulo de fase es igual a $45^{\circ}$ mediante la ecuación de cada recta. Las temperaturas de transición se resumen en la Tabla 5. 


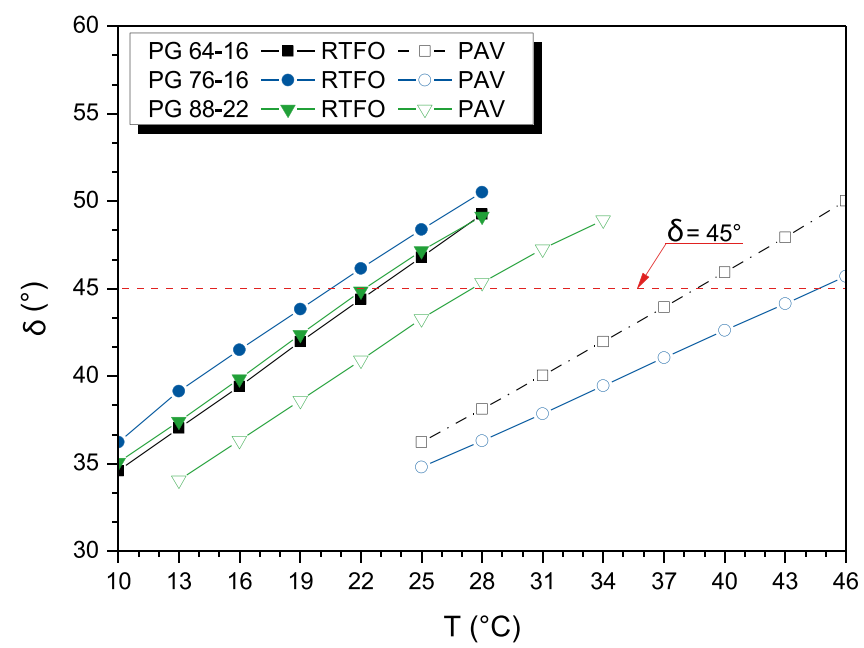

Figura 4. Determinación la temperatura de transición, RTF0 y PAV

De acuerdo a los resultados obtenidos se detectan dos consideraciones importantes: (1) la temperatura de transición en residuo de RTFO es inferior la temperatura de transición en residuo de PAV para los 3 ligantes, lo cual indica que un asfalto residuo de PAV comenzará a comportarse como un sólido viscoelástico a temperaturas mayores que un residuo de RTFO. Lo anterior confirma la teoría que un asfalto con mayor envejecimiento será más propenso a presentar agrietamiento por fatiga, ya que el fenómeno de fatiga se comenzará a presentar a partir de mayores temperaturas; sin embargo, lo anterior no implica que el asfalto con la mayor temperatura de transición presentará el peor comportamiento a la fatiga, simplemente se refiere a la temperatura máxima en la que comenzará su comportamiento sólido viscoelástico, el cual debe evaluarse con un ensayo de fatiga; (2) el PG 88 no presenta la misma tendencia que los otros ligantes después de envejecido en PAV, éste mantiene un punto de transición cercano al obtenido con residuo de RTFO, lo cual se puede atribuir a los químicos que forman parte de este asfalto para mezcla tibia.

Tabla 5. Temperatura de transición de los ligantes

\begin{tabular}{|c|c|c|}
\hline Ligante & Residuo RTFO & Residuo PAV \\
\hline PG 64-16 & $22.8^{\circ} \mathrm{C}$ & $38.5^{\circ} \mathrm{C}$ \\
\hline PG 76-16 & $20.8^{\circ} \mathrm{C}$ & $44.6^{\circ} \mathrm{C}$ \\
\hline PG 88-22 & $22.4^{\circ} \mathrm{C}$ & $27.9^{\circ} \mathrm{C}$ \\
\hline
\end{tabular}

La temperatura intermedia (calculada) del pavimento, mostrada en la Tabla 3, se indica como la temperatura de evaluación de la fatiga en los métodos PG y LAS. En el caso de los ligantes PG 64 y PG 76 se puede observar -en la Tabla 6- que dichas temperaturas se encuentran debajo la temperatura de transición con residuo de PAV, por lo que es apropiada para la evaluación a fatiga ya que el asfalto se encuentra en un estado sólido viscoelástico. Sin embargo, en el caso del PG 88 , la temperatura calculada de $37{ }^{\circ} \mathrm{C}$ es superior a su punto de transición $\left(27.9^{\circ} \mathrm{C}\right)$; por lo que evaluar la fatiga a la temperatura calculada podría arrojar resultados erróneos debido a que el asfalto se encontrará en un estado líquido viscoelástico. Lo anterior indica que la temperatura intermedia calculada no siempre puede ser adecuada para evaluar las propiedades de fatiga del ligante asfáltico.

Tabla 6. Comparativa de temperaturas para evaluar la fatiga

\begin{tabular}{|c|c|c|}
\hline Ligante & $\begin{array}{c}\text { Temperatura intermedia } \\
\text { calculada }\end{array}$ & $\begin{array}{c}\text { Temperatura de transición } \\
\text { PAV }\end{array}$ \\
\hline PG 64 & $28^{\circ} \mathrm{C}$ & $38.5^{\circ} \mathrm{C}$ \\
\hline PG 76 & $34^{\circ} \mathrm{C}$ & $44.6^{\circ} \mathrm{C}$ \\
\hline PG 88 & $37^{\circ} \mathrm{C}$ & $27.9^{\circ} \mathrm{C}$ \\
\hline
\end{tabular}

Observando los puntos de transición para los dos tipos de residuo, se decidió evaluar: (1) el ensayo LAS a 20 y $28^{\circ} \mathrm{C}$ con residuo de PAV, para obtener resultados debajo la temperatura de transición de los ligantes y compararlo con los resultados obtenidos con residuo de RTFO. En el caso de residuo con RTFO ya no se realizaron más evaluaciones debido a que a $20{ }^{\circ} \mathrm{C}$ ya se cuentan con resultados debajo la temperatura de transición para este tipo de residuo.

\section{EVALUACIÓN LAS PARA LA TEMPERATURA DE TRANSICIÓN}

Se realizó la evaluación del método LAS con el residuo de PAV para los tres ligantes, observando los resultados indicados en las Figuras 5 y 6 . De acuerdo a los resultados obtenidos, a $28^{\circ} \mathrm{C}$ el ligante PG 76 presenta una mayor resistencia a la fatiga, mientras que a $20{ }^{\circ} \mathrm{C}$ nuevamente se observa una discrepancia, ya que el mejor comportamiento a la fatiga se obtuvo entre dos materiales PG 64 y PG 88 de acuerdo al nivel de deformación aplicado.

\section{INFLUENCIA DEL ENVEJECIMIENTO}

En los resultados obtenidos se logró observar la influencia del envejecimiento de los asfaltos en la resistencia a fatiga de los mismos. En la Figura 7 se resumen los resultados del ensayo LAS para las temperaturas de 20 y $28{ }^{\circ} \mathrm{C}$ con los dos tipos de residuo (RTFO y PAV), donde se observa un comportamiento distinto para cada ligante, como se menciona a continuación:

- En el ligante PG 64 - a las dos temperaturas-después de envejecido en PAV se observa una mayor resistencia inicial y posteriormente una pérdida de la misma (mayor pendiente) en comparación de la resistencia con RTFO. Esto indica que 

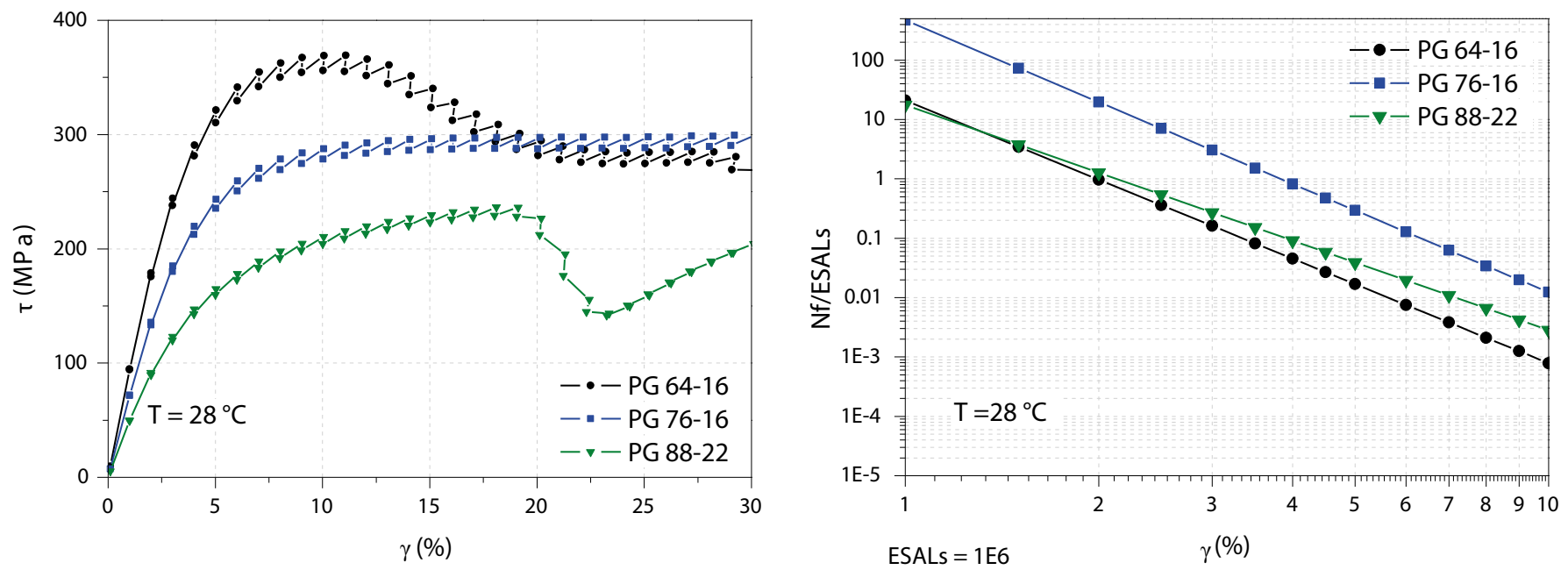

Figura 5. Resultados LAS a 28; PAV
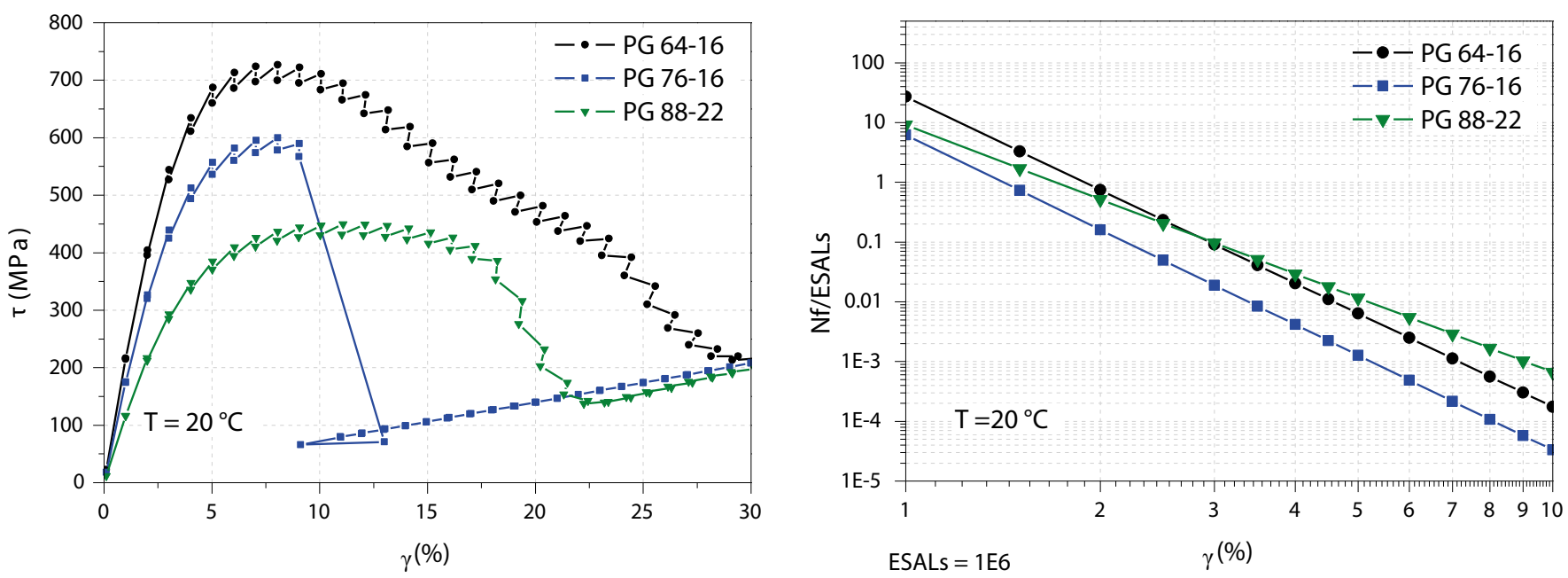

Figura 6. Resultados LAS a $20^{\circ} \mathrm{C}$; PAV

el asfalto después de PAV adquiere rigidez, pero a la vez pierde resistencia a la fatiga a mayor nivel de deformación.

- En el ligante PG 76 se observa un comportamiento similar, ya que las curvas conservan las mismas pendientes que el PG 64. Sin embargo, este ligante perdió resistencia después del envejecimiento en PAV a $20^{\circ} \mathrm{C}$, mientras que a $28^{\circ} \mathrm{C}$ sí se registra un incremento en la resistencia después de PAV. Este comportamiento es el que precisamente puede atribuirse a la fase o estado de la materia en el que se encuentre el asfalto.

- En el ligante PG 88 se observa un comportamiento similar tanto en residuo de RTFO y PAV, ya que las pendientes se conservan a ambas temperaturas; por lo que se determina que este asfalto no sufrió un cambio significativo en el comportamiento a la fatiga debido al proceso de envejecimiento.
De acuerdo a los resultados obtenidos, se deberán realizar validaciones en la mezcla asfáltica para definir tendencias y determinar cuál es la temperatura adecuada de evaluación de la fatiga en ligantes asfálticos, debido a los criterios que se han definido:

- Temperatura intermedia del pavimento (Tabla del Grado PG)

- Temperatura para $\mathrm{G}^{*} \operatorname{sen} \delta=5000 \mathrm{kPa}$

- Temperatura de transición del asfalto después de RTFO

- Temperatura de transición del asfalto después de PAV 

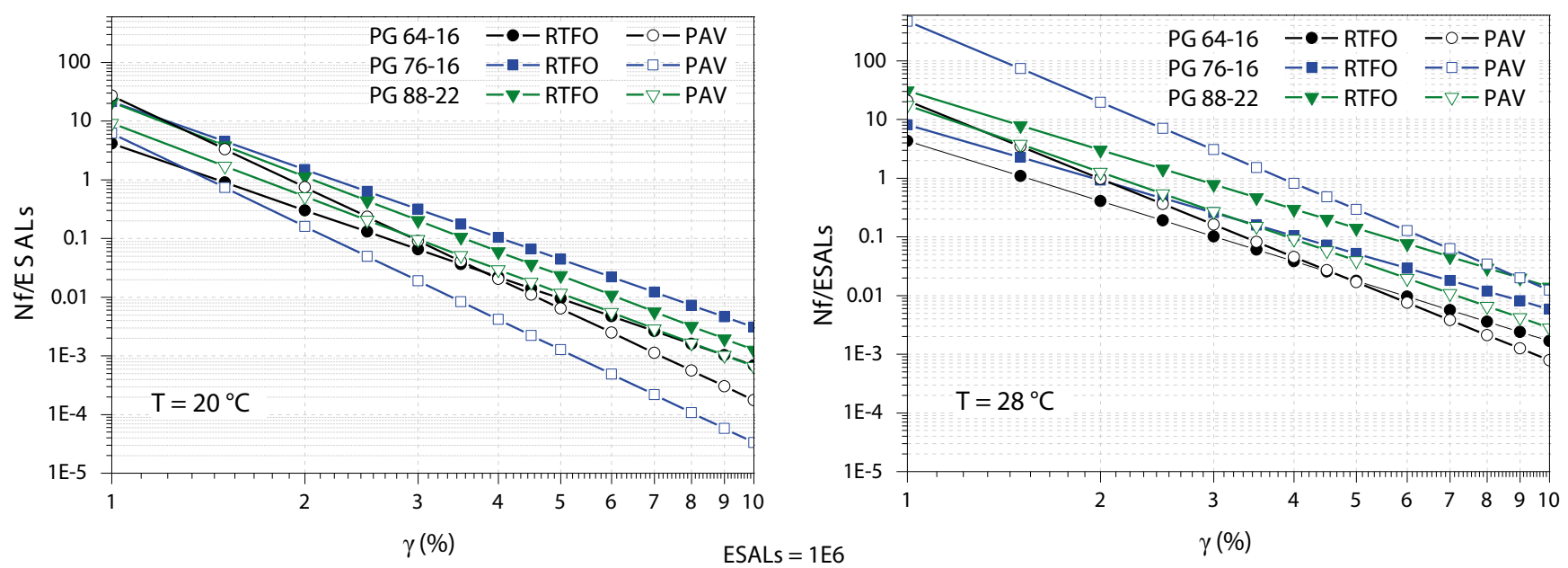

Figura 7. Comparación de residuos RTF0 y PAV, a 20 y $28^{\circ} \mathrm{C}$

Tabla 7. Resumen de desempeño para distintos criterios y evaluaciones

\begin{tabular}{|c|c|c|c|c|c|c|}
\hline \multirow{2}{*}{ Tipo de envejecimiento } & \multirow{2}{*}{$\begin{array}{c}\text { Criterio de selección de } \\
\text { temperatura }\end{array}$} & \multirow{2}{*}{$\begin{array}{c}\text { Temperatura del } \\
\text { ensayo }\end{array}$} & \multirow{2}{*}{ Tipo de Evaluación } & \multicolumn{3}{|c|}{ Desempeño } \\
\hline & & & & Superior & Medio & Inferior \\
\hline PAV & Temperatura intermedia & $28,34,37^{\circ} \mathrm{C}$ & PG & PG 88 & PG 76 & PG 64 \\
\hline \multirow{2}{*}{ RTFO } & Temperatura intermedia & $28,34,37^{\circ} \mathrm{C}$ & \multirow{4}{*}{ LAS } & PG 88 & PG 76 & PG 64 \\
\hline & $\begin{array}{c}\mathrm{G}^{*} \operatorname{sen} \delta=5000 \mathrm{kPa} \text { y Transición } \\
\text { RTFO }\end{array}$ & $20^{\circ} \mathrm{C}$ & & PG 76 & PG 88 & PG 64 \\
\hline \multirow{2}{*}{ PAV } & Transición PAV & $28^{\circ} \mathrm{C}$ & & PG 76 & PG 88 & PG 64 \\
\hline & $\mathrm{G}^{*} \operatorname{sen} \delta=5000 \mathrm{kPa}$ & $20^{\circ} \mathrm{C}$ & & PG 64 & PG 88 & PG 76 \\
\hline
\end{tabular}

\section{Comparación de criterios con 4PBB}

Se realizó una comparativa de desempeño entre los resultados obtenidos en la evaluación del ligante asfáltico y el ensayo a fatiga en mezclas asfálticas Viga a Flexión en 4 Puntos (4PBB, por sus siglas en inglés de Four Point Bending Beam). Para la fabricación de la mezcla se utilizó una granulometría densa con contenidos de asfalto: $5.4 \%, 5.3 \%$ y $5.9 \%$ para los ligantes PG 64-16, PG76-16 y PG88-22, respectivamente. El ensayo 4PBB se realizó en el modo de deformación controlada a una temperatura de $20{ }^{\circ} \mathrm{C}$ y una frecuencia de aplicación de la carga sinusoidal de $10 \mathrm{~Hz}$ a cuatro niveles de deformación: 300, 400, 500 y $650 \mu \varepsilon$. Las vigas fueron compactadas y cortadas para lograr unas dimensiones de $50 \mathrm{~mm}$ de altura x $63 \mathrm{~mm}$ de ancho y $380 \mathrm{~mm}$ de largo. El criterio de falla para el análisis de resultados fue el criterio clásico: reducción del $50 \%$ del valor del módulo de rigidez inicial. La Figura 8 presenta los resultados obtenidos en los ensayos de fatiga en viga a flexión en cuatro puntos.

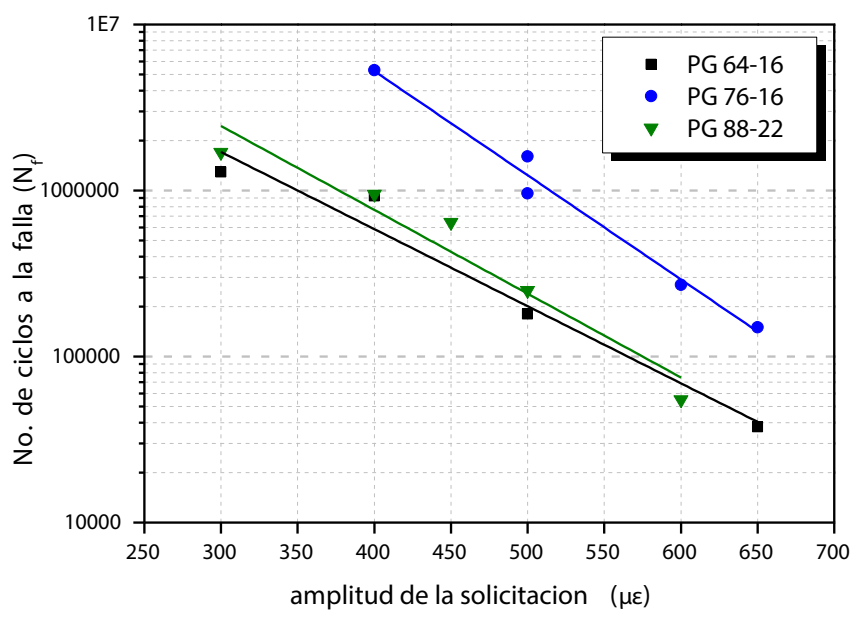

Figura 8. Curva de Wöhler en 4PBB

De acuerdo a la curva de Wöhler de las mezclas asfálticas se puede determinar que el PG 76 se desempeñó mejor que el PG 88 y PG 64. Comparando estos resultados con el resumen de la Tabla 7 se puede definir que el criterio, que correlaciona el desempeño de la 
mezcla asfáltica con el asfalto, es la temperatura de transición en el ensayo LAS.

Aunado a lo anterior, el ensayo a $20{ }^{\circ} \mathrm{C}$ con residuo de PAV en el ensayo LAS también se encuentra en un rango debajo la temperatura de transición y los resultados obtenidos no fueron congruentes con lo mencionado anteriormente. Esto se puede atribuir a que un asfalto residuo de PAV a una temperatura de $20{ }^{\circ} \mathrm{C}$ es muy rígido, lo que puede generar una disminución en la adherencia asfalto-plato provocando dispersiones en el ensayo.

\section{CONCLUSIONES}

- La caracterización por Grado PG evalúa la resistencia a fatiga de un ligante asfáltico limitando su rigidez a la temperatura intermedia del pavimento. Sin embargo, de acuerdo a diversas investigaciones, dicha evaluación no considera el efecto del daño acumulado en el ligante ya que lo evalúa en el rango viscoelástico lineal, además de fijar la temperatura del pavimento a un valor calculado.

- El ensayo LAS es congruente con el Grado PG cuando el asfalto se encuentra en un estado líquido viscoso (superior al punto de transición). Debajo la temperatura de transición ya existen discrepancias en los resultados.
- La temperatura intermedia del pavimento indicada en el Grado PG no siempre se encuentra debajo la temperatura de transición del asfalto residuo de PAV, para poder garantizar un estado sólido viscoelástico; por lo que el criterio del Grado PG podría no ser el adecuado cuando se utilicen asfaltos con distintos tipos de modificadores.

- La temperatura de transición es donde el ligante comienza a entrar en un estado sólido viscoelástico y se podría considerar como la temperatura máxima para evaluar la resistencia a fatiga de un ligante.

- Se observaron cambios sustanciales con la variación de la temperatura, ya que con un mismo tipo de residuo (RTFO o PAV), la respuesta de los ligantes fue completamente distinta. Se demuestra que la temperatura juega un rol muy importante en la resistencia del ligante a la fatiga, por lo cual es indispensable determinar un parámetro que defina la temperatura adecuada de los ensayos de fatiga en ligantes asfálticos.

- Se deberán continuar las validaciones al ensayo LAS a distintas temperaturas para determinar si el punto de transición es un parámetro adecuado fijar la temperatura máxima de ensayo de fatiga en ligantes asfálticos.

\section{REFERENCIAS}

1. ASTM D6373 (2008). Standard Specification for Performance Graded Asphalt Binder. Obtenido de ASTM International: http:// www.astm.org/Standards/ D6373.htm

2. ASTM D7175 (2008). Standard Test Method for Determining the Rheological Properties of Asphalt Binder Using a Dynamic Shear Rheometer. Obtenido de ASTM International: http://www.astm.org/Standards/D7175.htm

3. AASHTO TP101 (2014). Estimating Damage Tolerance of Asphalt Binders Using the Linear Amplitude Sweep. Obtenido de Modified Asphalt Research Center: http://uwmarc.wisc.edu/files/linearamplitudesweep/AASHTO-TP101-LAS-May-2013-v2.pdf

4. Bahia, H., Hanson, D., Zeng, M., Zhai, H., Khatri, M., \& Anderson, R. (2001). NCHRP Report 459: Characterization of modified asphalt binders in Superpave Mix Design. Washington D.C., EUA: National Academy Press.

5. Delgado, H., Ayala, Y. \& Garnica, P. (2015). Evaluación de la fatiga en ligantes asfálticos. XVIII Congreso Ibero Latinoamericano del Asfalto. Bariloche, Argentina.

6. Ghuzlan, K. \& Carpenter, S. (2003). Traditional Fatigue Analysis of Asphalt Concrete Mixtures. TRB 2003 Annual Meeting. Obtenido de LTRC: http://www. ltrc.lsu.edu/TRB_82/TRB2003-002146.pdf

7. Gutiérrez, Á. (2013). Reducción de la fisuración por fatiga de la mezcla asfáltica por medio del control la temperatura de transición del asfalto. XVII Congreso Ibero-Latinoamericano del Asfalto. Guatemala.

8. Hintz, C., Velasquez, R., Johnson, C. \& Bahia, H. (2013). Modification and Validation of Linear Amplitude Sweep Test for Binder Fatigue Specification. Transportation Research Board, Vol. 2207. E.U.A.

9. Hossein, A., Leek, C. \& Nikraz, H. (2013). Effect of temperature on fatigue life of asphalt mixture. 15th Australian Asphalt Pavement Association International Flexible Pavements Conference. Obtenido de HAD: http://www.h-a-d.hr/pubfile.php?id=762

10. Van Rompu, J., Di Benedetto, H., Buannic, M., Gallet \& T., Ruot, C. (2012). New fatigue test on bituminous binders: Experimental results and modeling. Construction and Building Materials, 37, 19 